\title{
Probing the tidal disruption flares of massive black holes with high-energy neutrinos
}

\author{
Xiang-Yu Wang ${ }^{1,2}$, Ruo-Yu Liu ${ }^{1,2}$, Zi-Gao Dai ${ }^{1,2}$ and K. S. Cheng ${ }^{3}$ \\ ${ }^{1}$ Department of Astronomy, Nanjing University, Nanjing 210093, China \\ ${ }^{2}$ Key laboratory of Modern Astronomy and Astrophysics (Nanjing University), Ministry of Education, Nanjing 210093, China \\ ${ }^{3}$ Department of Physics, University of Hong Kong, Hong Kong, China.
}

\begin{abstract}
The recently discovered high-energy transient Swift J164449.3+573451 (Sw J1644+57) is thought to arise from the tidal disruption of a passing star by a dormant massive black hole. Modeling of the broadband emission suggests the presence of a powerful relativistic jet, which contributes dominantly to the observed X-ray emission. Here we suggest that protons can be accelerated to ultra-high energies by internal shocks occurring in the jets, but their flux is insufficient to account for the observed flux of ultra-high energy cosmic rays. High energy protons can produce $\sim 0.1$ $10 \mathrm{PeV}$ neutrinos through photomeson interactions with X-ray photons. The large X-ray fluence $\left(7 \times 10^{-4} \mathrm{erg} \mathrm{cm}^{-2}\right)$ and high photopion efficiency, together with the insignificant cooling of secondary mesons, result in bright neutrino emission expected from $\mathrm{Sw} \mathrm{J} 1644+57$ if the jet composition is matter-dominated. One to several neutrinos may be detected by a $\mathrm{Km}^{3}$-scale detector from one tidal disruption event similar to Sw J1644+57, thereby providing a powerful probe of the composition of the jets.
\end{abstract}

PACS numbers: 95.85Ry, 98.70Qy, 98.70Sa

Massive black holes are believed to reside at the centers of most galaxies and the vast majority of them are considered to be dormant. It was long predicted that, if a star of mass $M_{\star}$ and radius $R_{\star}$ passes occasionally within the disruption radius $r_{T} \simeq R_{\star}\left(M_{\mathrm{BH}} / M_{\star}\right)^{1 / 3}$ of the dormant black hole (where $M_{\mathrm{BH}}$ is the black hole mass), the star will be torn apart by gravitational tidal forces, leading to a transient accretion disk and a bright panchromatic flare [1]. Candidates of such tidal disruption flares (TDFs) have been suggested[2]. Relativistic jets are expected to form in such accretion disk system and may produce observational phenomena as well [3].

The high-energy transient Swift J164449.3+573451 (hereafter Sw J1644+57) was discovered by the Swift satellite at 2011 March 28 12:57:45 UT[4]. The facts that it occurred near the center of a galaxy, no archival X-ray emission before detection and its analogy with blazars in the X-ray emission suggest that it is mostly likely to be a TDF event [5 7]. At redshift $z=0.3534$ (corresponding to a luminosity distance of $d_{L}=1.8 \mathrm{Gpc}$ ) [6], the early X-ray flare emission reached a peak luminosity of $L_{\mathrm{X}} \simeq 4.3 \times 10^{48} \mathrm{erg} \mathrm{s}^{-1}$ (isotropic equivalent) and then transits to a low state with a median luminosity of $L_{\mathrm{X}} \simeq 2.96 \times 10^{47} \mathrm{erg} \mathrm{s}^{-1}$ in $0.4-13.5 \mathrm{keV}$ over a time $\Delta T \sim 10^{6} \mathrm{~s}[4]$. Correcting for the live-time fraction of the observation, the total unabsorbed fluence is $S_{\mathrm{X}}=7.1 \times 10^{-4} \mathrm{erg} \mathrm{cm}^{-2}$ in the observed $0.3-10.0 \mathrm{keV}$ band [4]. This gives an estimate of the total isotropic equivalent energy of $E_{\mathrm{X}}=3 \times 10^{53} \mathrm{erg}$ in the $0.4-13.5$ $\mathrm{keV}$ rest frame energy band. The observed minimum X-ray variability time of $\mathrm{Sw} \mathrm{J} 1644+57, t_{v} \simeq 100 \mathrm{~s}[4]$, constrains the size of the black hole under the assumption that the size of the central engine determines the shortest variability and suggest an upper limit on the massive black hole mass $M_{\mathrm{BH}} \leq 8 \times 10^{6} M_{\odot}$. The observed peak luminosity is super-Eddington and requires a strongly anisotropic radiation pattern with a relativis- tic jet of a bulk Lorentz factor of $\Gamma \simeq 10$ pointed towards us [4, 5]. Modeling of the broadband spectral energy distribution (SED) also requires a powerful relativistic jet with $\Gamma \simeq 10$, which produce dominantly the observed $\mathrm{X}$ ray emission [4, 5]. A relativistic jet is also required to explain the radio transient [8]. However, how the jet is launched is not well-understood. It is believed that the composition of the jet, whether it is matter-dominated or magnetic field-dominated, is crucial to unveiling the formation mechanism of the jets. In this paper, we suggest that jets in TDFs can produce bright emission in highenergy neutrinos if the jet is matter-dominated, and thus neutrino observations provide an important tool to diagnose the jet composition.

Internal shocks and particle acceleration. - We consider that a TDF event produces a relativistic matterdominated wind of luminosity $L_{w} \sim 10^{49} \mathrm{erg} \mathrm{s}^{-1}$, moving with a bulk Lorentz factor $\Gamma \sim 10$. Variability of the source on time scale $t_{v}$, resulting in fluctuations in the wind bulk Lorentz factor $\Gamma$ on similar time scale, would lead to semi-relativistic internal shocks $[9]$ in the ejecta at a radius

$$
R \simeq 2 \Gamma^{2} c t_{v}=6 \times 10^{14} \Gamma_{1}^{2} t_{v, 2} \mathrm{~cm},
$$

which is well above the photosphere radius at $R_{p h}=$ $\sigma_{\mathrm{T}} L_{w} /\left(4 \pi \Gamma^{3} m_{p} c^{3}\right)=1.2 \times 10^{13} L_{w, 49} \Gamma_{1}^{-3} \mathrm{~cm}$, where $\sigma_{\mathrm{T}}$ is the Thomson cross section. We use c.g.s. units and the denotation $Q=10^{x} Q_{x}$ throughout the paper.

Denoting $\epsilon_{B}$ as the fraction of the wind kinetic energy converted into magnetic fields, we have a magnetic field $B^{\prime}=\left(8 \pi \epsilon_{B} L_{w} / 4 \pi R^{2} \Gamma^{2} c\right)^{1 / 2}=$ $1.3 \times 10^{3} \epsilon_{B,-1}^{1 / 2} L_{w, 49}^{1 / 2} R_{14.8}^{-1} \Gamma_{1}^{-1} \mathrm{G}$, where the prime symbol represents quantities measured in the comoving frame of the shock. It is assumed that internal shocks accelerate protons with a spectrum $d n / d \varepsilon_{p} \sim \varepsilon_{p}^{-2}$, where $\varepsilon_{p}$ is the proton energy in the observer frame. The maximum proton energy is set by comparing the acceleration time 
$t_{\mathrm{acc}}^{\prime}=\alpha \varepsilon_{p} /\left(e \Gamma B^{\prime} c\right)=860 \alpha\left(\frac{\varepsilon_{p}}{10^{20} \mathrm{eV}}\right) \epsilon_{B,-1}^{-1 / 2} L_{w, 49}^{-1 / 2} R_{14.8} \mathrm{~s}$ with the shock dynamic time $t_{\text {dyn }}^{\prime}=R / \Gamma c=10^{3} \Gamma_{1} t_{v, 2} \mathrm{~s}$, where $\alpha \sim$ a few, describing the ratio between the acceleration time and Larmor time. This gives a maximum proton energy of $\varepsilon_{\max , \mathrm{dyn}}=2.4 \times 10^{20} \alpha^{-1} \epsilon_{B,-1}^{1 / 2} L_{w, 49}^{1 / 2} \Gamma_{1}^{-1} \mathrm{eV}$. The maximum energy is also limited by the cooling time of protons. The synchrotron cooling time is $t_{\text {syn }}^{\prime}=6 \pi m_{p}^{4} c^{3} \Gamma /\left(\sigma_{T} m_{e}^{2} \varepsilon_{p} B^{2}\right)=$ $240 \epsilon_{B,-1}^{-1} L_{w, 49}^{-1} R_{14.8}^{2} \Gamma_{1}^{3}\left(\frac{\varepsilon_{p}}{10^{20} \mathrm{eV}}\right)^{-1} \mathrm{~s}$, which gives a maximum proton energy $\varepsilon_{\text {max }, \mathrm{syn}}=0.5 \times$ $10^{20} \alpha^{-1 / 2} \epsilon_{B,-1}^{-1 / 4} L_{w, 49}^{-1 / 4} t_{v, 2}^{1 / 2} \Gamma_{1}^{5 / 2} \mathrm{eV}$. Another process that may prohibit the acceleration of protons to ultrahigh (UHE) energies is the photopion cooling loss. UHE protons of energy $\varepsilon_{p}$ interact with soft photons with energy $\epsilon_{\gamma}=0.15 \mathrm{GeV}^{2} \Gamma^{2} / \varepsilon_{p}=0.15 \Gamma_{1}^{2} \varepsilon_{p, 20}^{-1} \mathrm{eV}$, which locate at near infrared (NIR) band for typical jet parameters. The number density of NIR photons in the comoving frame is $n_{\mathrm{NIR}}^{\prime}=L_{\mathrm{NIR}} /\left(4 \pi R^{2} \Gamma c \epsilon_{\gamma}\right)=$ $2.5 \times 10^{14} L_{\mathrm{NIR}, 44} \Gamma_{1}^{-5} t_{v, 2}^{-2}\left(\epsilon_{\gamma} / 0.15 \mathrm{eV}\right)^{-1} \mathrm{~cm}^{-3}$, where $L_{\mathrm{NIR}} \simeq 10^{44} \mathrm{ergs}^{-1}$ is the NIR luminosity at times a few days after the initial trigger [4]. The photopion cooling time in the comoving frame is $t_{p \gamma}^{\prime}=$ $1 /\left(\sigma_{p \gamma} n_{\mathrm{NIR}}^{\prime} c K_{p \gamma}\right)=1200 L_{\mathrm{NIR}, 44}^{-1} \Gamma_{1}^{5} t_{v, 2}^{2}\left(\epsilon_{\gamma} / 0.15 \mathrm{eV}\right) \mathrm{s}$, where $K_{p \gamma}$ is the inelasticity and $\sigma_{p \gamma}=5 \times 10^{-28} \mathrm{~cm}^{-2}$ is the peak cross section at the $\Delta$ resonance. By equating $t_{a c c}^{\prime}$ with $t_{p \gamma}^{\prime}$, we get the maximum proton energy limited by the photopion cooling process, $\varepsilon_{\max , \mathrm{p} \gamma}=1.3 \times 10^{20} \alpha^{-1} \epsilon_{B,-1}^{1 / 4} L_{w, 49}^{1 / 4} L_{\mathrm{NIR}, 44}^{-1 / 2} \Gamma_{1}^{5 / 2} t_{v, 2}^{1 / 2} \mathrm{eV}$. Thus, internal shocks in TDFs can accelerate protons to energies above $10^{19} \mathrm{eV}$, in support of the earlier suggestion that TDFs can produce ultra-high energy cosmic rays (UHECRs) [10]. However, the flux of such UHE protons contributed by TDFs in the universe is insufficient to explain the observed flux of UHECRs, as we show below.

UHECR flux - The Swift BAT, with a field of view of $S_{\mathrm{FOV}} \simeq 4 \pi / 7 \mathrm{sr}$, has detected one such event in a time $T \simeq 7$ years at a flux that would have been detectable up to a luminosity distance of $d_{\max }=5 \mathrm{Gpc}$ [4]. Therefore we will assume that the rate of Sw J1644+57-like event is

$$
\dot{R}=\frac{4 \pi}{S_{\mathrm{FOV}}} \frac{1}{T} \frac{1}{(4 / 3) \pi d_{\max }^{3}} \simeq 2 \times 10^{-12} \mathrm{Mpc}^{-3} \mathrm{yr}^{-1}
$$

and the energy injection rate in X-rays is $\dot{\varepsilon}_{\mathrm{X}}=\dot{R} E_{\mathrm{X}}=$ $6 \times 10^{41} \mathrm{ergMpc}^{-3} \mathrm{yr}^{-1}$. Following ref.[11], the total energy in accelerated protons $E_{p}$ can be parameterized by $E_{p}=\xi_{\mathrm{p}} E_{\mathrm{X}}$, where the non-thermal baryon loading factor $\xi_{\mathrm{p}}$ can be expressed by $\xi_{\mathrm{p}}=10 \varsigma_{p} \eta_{e}^{-1}\left(0.1 / \epsilon_{e}\right), \epsilon_{e}$ is the fraction of the shock internal energy that goes into nonthermal electrons, $\eta_{e}$ is the radiative efficiency of these electrons, and $\varsigma_{p}$ is the proton acceleration efficiency. Modeling of the afterglows of gamma-ray bursts gives a typical value $\epsilon_{e} \simeq 0.1$ for relativistic shocks [12], so the typical value of $\xi_{\mathrm{p}}$ would be $\sim 10$. Thus the differential energy injection rate in protons is

$\varepsilon_{p}^{2} \frac{d \dot{n}}{d \varepsilon_{p}}=\frac{\xi_{\mathrm{p}} \dot{R} E_{\mathrm{X}}}{\ln \left(\varepsilon_{p, \text { max }} / \varepsilon_{p, \text { min }}\right)} \simeq 6 \times 10^{41} \xi_{\mathrm{p}, 1} \mathrm{ergMpc}^{-3} \mathrm{yr}^{-1}$,

where $\varepsilon_{p, \max }$ and $\varepsilon_{p, \min }$ are, respectively, the maximum and minimum energy of accelerated protons and we have used $\ln \left(\varepsilon_{p, \max } / \varepsilon_{p, \min }\right)=10$ in the last step. For $\xi_{\mathrm{p}}$ of the order $\sim 10$, this rate is much smaller than the required energy generation rate of cosmic rays per energy decade from $0.5-20 \times 10^{44} \mathrm{ergMpc}^{-3} \mathrm{yr}^{-1}$ deduced by different authors [13]. Note, however, that it was only the presence of short, powerful bursts early on that alerted us to its presence, so we can not exclude the possibility that many other similar, but rather less variable, events could be undetected.

Pion production. - Now we consider the neutrino emission produced by these protons interacting with the soft photons in the sources. Protons lose $\sim 20 \%$ of their energy at each $p \gamma$ interaction, dominated by the $\Delta$ resonance. Approximately half of the pions are charged and decay into high energy neutrinos $\pi^{+} \rightarrow \mu^{+}+\nu_{\mu} \rightarrow$ $e^{+}+\nu_{e}+\bar{\nu}_{\mu}+\nu_{\mu}$, with the energy distributed roughly equally among the decay products. The fraction of energy lost by protons to pions is $f_{p \gamma}=R /\left(\Gamma c t_{p \gamma}^{\prime}\right)$. Denoting by $n\left(\epsilon_{\gamma}\right) d \epsilon_{\gamma}$ the number density of photons in the energy range $\epsilon_{\gamma}$ to $\epsilon_{\gamma}+d \epsilon_{\gamma}$, the cooling time of protons in the shock comoving frame for $p \gamma$ process is given by

$$
t_{p \gamma}^{\prime}=\frac{c}{2 \Gamma_{p}^{2}} \int_{\epsilon_{t h}}^{\infty} d \epsilon \sigma(\epsilon) K(\epsilon) \epsilon \int_{\epsilon / 2 \Gamma_{p}}^{\infty} d x x^{-2} n(x),
$$

where $\Gamma_{p}=\varepsilon_{p} / \Gamma m_{p} c^{2}, \sigma$ and $K$ are respectively the cross section and the inelasticity for $p \gamma$ process [14]. The spectrum from infrared to X-ray frequencies of $\mathrm{Sw}$ $\mathrm{J} 1644+57$ can be approximately described by a broken power-law with $n\left(\epsilon_{\gamma}\right)=n_{b}\left(\epsilon_{\gamma} / \epsilon_{b}\right)^{-\alpha}$ for $\epsilon_{\gamma}<\epsilon_{b}$ and $n\left(\epsilon_{\gamma}\right)=n_{b}\left(\epsilon_{\gamma} / \epsilon_{b}\right)^{-\beta}$ for $\epsilon_{\gamma}>\epsilon_{b}$, where $\epsilon_{b} \sim 1 \mathrm{KeV}$, $\alpha \simeq 2 / 3$ and $\beta \simeq 2[4]$. Approximating the integral by the contribution from the resonance we obtain

$$
f_{p \gamma}\left(\varepsilon_{p}\right) \simeq 0.35 \frac{L_{\mathrm{X}, 47.5}}{\Gamma_{1}^{4} t_{v, 2} \epsilon_{\mathrm{b}, \mathrm{KeV}}}\left\{\begin{array}{l}
\left(\varepsilon_{p} / \varepsilon_{p b}\right)^{\beta-1}\left(\varepsilon_{p}<\varepsilon_{p b}\right) \\
\left(\varepsilon_{p} / \varepsilon_{p b}\right)^{\alpha-1}\left(\varepsilon_{p}>\varepsilon_{p b}\right)
\end{array}\right.
$$

where $\varepsilon_{p b}=0.15 \mathrm{GeV}^{2} \Gamma^{2} / \epsilon_{b}=1.5 \times 10^{16} \Gamma_{1}^{2} \epsilon_{\mathrm{b}, \mathrm{KeV}}^{-1} \mathrm{eV}$ is the proton break energy. To include the effect of multipion production and high inelasticity (which increases from $\simeq 0.2$ at energies not far above the threshold to $\sim 0.5-0.6$ at energies where multi-pion production dominates) at high energies 15, 16], the above estimate of $f_{p \gamma}$ should be multiplied by factor of $\sim 2$. As the neutrino energy is $\sim 5 \%$ of the proton energy, the neutrino flux will peak at $\varepsilon_{\nu b} \simeq 7.5 \times 10^{14} \Gamma_{1}^{2} \epsilon_{\mathrm{b}, \mathrm{KeV}}^{-1} \mathrm{eV}$.

In the modeling of the SED of Sw J1644+57, upper limits from Fermi and VERITAS require $\Gamma<20$ in the $\mathrm{X}$-ray emitting region [4]. It is useful to express $f_{p \gamma}$ as a function of the pair production optical depth $\tau_{\gamma \gamma}$. The optical depth for pair production of a photon of energy $\varepsilon_{h}$ is $\tau_{\gamma \gamma}\left(\epsilon_{h}\right)=\frac{R}{\Gamma l_{\gamma \gamma}}=\frac{R}{\Gamma} \frac{\sigma_{T}}{16} \frac{U_{\gamma} \epsilon_{h}}{\Gamma\left(m_{e} c^{2}\right)^{2}}$, where $l_{\gamma \gamma}$ is the mean 
free path. The fraction of energy lost by protons to pions is $f_{p \gamma} \simeq \frac{R}{\Gamma} \frac{U_{\gamma}}{2 \varepsilon_{p}^{\prime}} \sigma_{p \gamma} K_{p \gamma}\left(\varepsilon_{p} / \varepsilon_{p b}\right)^{\alpha-1}$ for protons with energy $\varepsilon_{p}>\varepsilon_{p b}$. Thus, we have 17.

$$
f_{p \gamma}\left(\varepsilon_{p}\right) \simeq 0.5 \tau_{\gamma \gamma}(100 \mathrm{MeV})\left(\frac{\varepsilon_{p b}}{1.5 \times 10^{16} \mathrm{eV}}\right)\left(\frac{\varepsilon_{p}}{\varepsilon_{p b}}\right)^{\alpha-1} .
$$

Modeling of the SED of Sw J1644+57 requires $\tau_{\gamma \gamma}(100 \mathrm{MeV})>1$ [4], so we conclude that a significant fraction $(>50 \%)$ of the energy of protons accelerated to energies larger than the break energy, $\varepsilon_{p b} \sim 10^{16} \mathrm{eV}$, would be lost to pion production.

Meson cooling. - The neutrino production efficiency will be reduced if the secondary mesons suffer from cooling before decaying to neutrinos and other products [18]. The pions and muons suffer from radiative cooling due to both synchrotron emission and inverseCompton emission. The total radiative cooling time for pions is $t_{\pi, \text { rad }}^{\prime}=3 m_{\pi}^{4} c^{3} /\left[4 \sigma_{T} m_{e}^{2} \epsilon_{\pi}^{\prime} U_{B}^{\prime}\left(1+f_{\mathrm{IC}}\right)\right] \simeq$ $2 \times 10^{6}\left(\epsilon_{\pi}^{\prime} / 1 \mathrm{TeV}\right)^{-1} \epsilon_{B,-1}^{-1} L_{w, 48}^{-1} R_{14.5}^{2} \Gamma_{1}^{2} \mathrm{~s}$, where $U_{B}^{\prime}$ is the energy density of the magnetic filed in the shock region and $f_{\mathrm{IC}} \leq 1$ is the correction factor accounting for the inverse-Compton loss. By comparing this cooling time $t_{\pi, \text { rad }}^{\prime}$ with the lifetime of pions $\tau_{\pi}^{\prime}=\gamma_{\pi} \tau=$ $1.9 \times 10^{-4}\left(\epsilon_{\pi}^{\prime} / 1 \mathrm{TeV}\right) \mathrm{s}$ in the shock comoving frame, where $\gamma_{\pi}$ and $\tau$ are the pion Lorentz factor and proper lifetime, one can find a critical energy (in the observer frame) for pions, above which the effect of radiative cooling starts to suppress the neutrino flux,

$$
\varepsilon_{\pi, \text { rad }}=6.3 \times 10^{5} \epsilon_{B,-1}^{-1 / 2} L_{w, 49}^{-1 / 2} \Gamma_{1}^{4} t_{v, 2} \mathrm{TeV} .
$$

Similarly, by comparing the radiative cooling time $t_{\mu, \text { rad }}^{\prime}$, with the lifetime of muons $\tau_{\mu}^{\prime}$, one can obtain a critical energy for muons, above which the effect of radiative cooling starts to suppress the anti-muon neutrino flux,

$$
\varepsilon_{\mu, \text { rad }}=3.2 \times 10^{4} \epsilon_{B,-1}^{-1 / 2} L_{w, 49}^{-1 / 2} \Gamma_{1}^{4} t_{v, 2} \mathrm{TeV} .
$$

The above estimates lead us to conclude that the neutrino flux below $\sim 10^{16} \mathrm{eV}$ is not affected by the meson cooling for typical parameters of TDFs. At higher energies, however, pion cooling and muon cooling will suppress the neutrino flux by a factor approximately given, respectively, by [19]

$$
\zeta_{\pi}=\operatorname{Min}\left\{t_{\pi, \mathrm{rad}}^{\prime} / \tau_{\pi}^{\prime}, 1\right\}, \zeta_{\mu}=\operatorname{Min}\left\{t_{\mu, \mathrm{rad}}^{\prime} / \tau_{\mu}^{\prime}, 1\right\} .
$$

The spectrum and flux of the neutrino flare. - The fluence spectrum of the muon neutrino $\left(\nu_{\mu}+\bar{\nu}_{\mu}\right)$ emission from one TDF is

$\varepsilon_{\nu}^{2} \Phi_{\nu}=\varepsilon_{p}^{2} \frac{d n_{p}}{d \varepsilon_{p}} \frac{f_{p \gamma} \zeta_{\pi}\left(1+\zeta_{\mu}\right)}{8}=\frac{E_{\mathrm{X}}}{32 \pi d_{L}^{2}} \frac{\xi_{\mathrm{p}} f_{p \gamma} \zeta_{\pi}\left(1+\zeta_{\mu}\right)}{\ln \left(\varepsilon_{p, \max } / \varepsilon_{p, \min }\right)}$,

where $\varepsilon_{p}^{2} \frac{d n_{p}}{d \varepsilon_{p}}=E_{p} /\left[4 \pi d_{L}^{2} \ln \left(\varepsilon_{p, \max } / \varepsilon_{p, \text { min }}\right)\right]$ is the differential proton fluence produced by one TDF of total energy $E_{p}=\xi_{\mathrm{p}} E_{\mathrm{X}}$ in protons and the factor $1 / 8$ represents that the neutrinos produced by pion decay carry

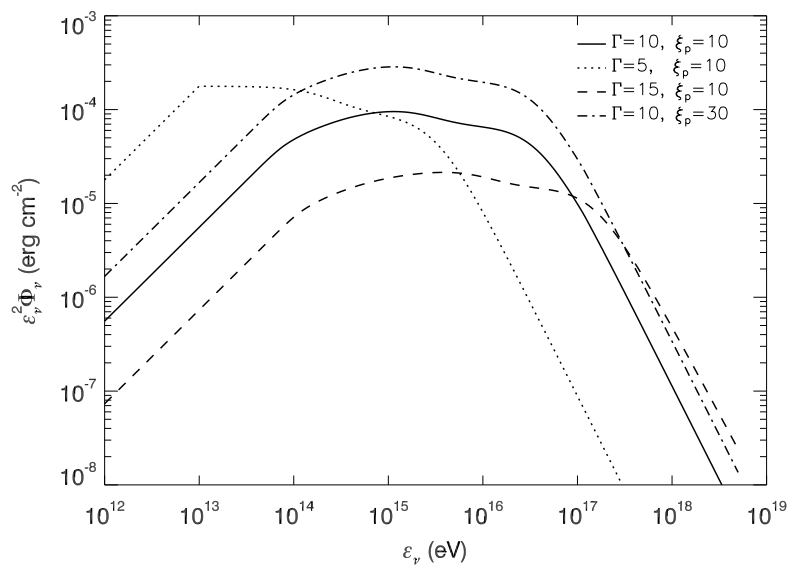

FIG. 1: The expected muon neutrino $\left(\nu_{\mu}+\bar{\nu}_{\mu}\right)$ spectra from Sw J1644+57 for different jet parameters. In all lines, $S_{\mathrm{X}}=$ $7 \times 10^{-4} \mathrm{ergcm}^{-2}, L_{\mathrm{X}}=3 \times 10^{47} \mathrm{ergs}^{-1}, t_{v}=100 \mathrm{~s}, \epsilon_{b}=1 \mathrm{KeV}$ and $\epsilon_{B}=0.1$ are used.

one-eighth of the energy lost by protons to pion production, since charged and neutral pions are produced with roughly equal probability and muon neutrinos carry roughly one-fourth of the pion energy in pion decay. Fig.1 shows the expected muon neutrino fluence spectra from Sw J1644+57, obtained by using the Lorentzian form for the photopion production cross section at the resonance peak plus a component contributed by multi-pion production at higher energies [20] in calculating $t_{p \gamma}^{\prime}$ with Eq.(4). The initial rise in the spectrum at low energies is caused by the increasing pion production efficiency with energy, while the mild steepening and sharp steepening seen at higher energies are caused by muon cooling and pion cooling respectively.

Now we estimate the number of neutrinos that can be detected from one TDF event similar to $\mathrm{Sw}$ $\mathrm{J} 1644+57$. The detection efficiency in water or ice of ultra-relativistic upward-going muon neutrinos with energies $\varepsilon_{\nu}$ is $P_{\nu \mu} \simeq 7 \times 10^{-5}\left(\varepsilon_{\nu} / 10^{4.5} \mathrm{GeV}\right)^{\kappa}$, where $\kappa=1.35$ for $\varepsilon_{\nu}<10^{4.5} \mathrm{GeV}$, and $\kappa=0.55$ for $\varepsilon_{\nu}>$ $10^{4.5} \mathrm{GeV}[21]$. For neutrino fluence spectrum parameterized by $\varepsilon_{\nu}^{2} \Phi_{\nu}$, the number of $\nu_{\mu}$ and $\bar{\nu}_{\mu}$ above a certain energy $\varepsilon_{\nu 0}$ detected by a $\mathrm{km}^{3}$-scale neutrino detector, such as IceCube, with area $A=10^{10} A_{10} \mathrm{~cm}^{2}$ is

$$
\begin{aligned}
& N_{\nu}\left(>\varepsilon_{\nu 0}=10^{4.5} \mathrm{GeV}\right)=\int_{\varepsilon_{\nu 0}}^{\infty} \Phi_{\nu} P_{\nu \mu} A d \varepsilon_{\nu} \\
& \simeq 2 \xi_{\mathrm{p}, 1} A_{10} f_{p \gamma}\left(\varepsilon_{p b}\right)\left(\frac{E_{X}}{3 \times 10^{53} \mathrm{erg}}\right)\left(\frac{d_{L}}{1.8 \mathrm{Gpc}}\right)^{-2},
\end{aligned}
$$

where we have used $\ln \left(\varepsilon_{p, \max } / \varepsilon_{p, \min }\right)=10, \zeta_{\pi} \simeq 1, \zeta_{\mu} \simeq$ $1, \alpha=2 / 3$ and $\beta=2$ in the last step. As $f_{p \gamma}>0.5$, we expect $\geq 1$ neutrinos above $30 \mathrm{TeV}$ could be detected from $\mathrm{Sw} 1644+57$ by $\mathrm{Km}^{3}$-scale detectors for $\xi_{\mathrm{p}}=10$. A careful calculation of the number of neutrinos above a certain energy as a function of the neutrino energy is shown in Fig. 2. A lower bulk Lorentz factor or a higher wind luminosity (i.e. a larger $\xi_{\mathrm{p}}$ ) leads to a larger number 


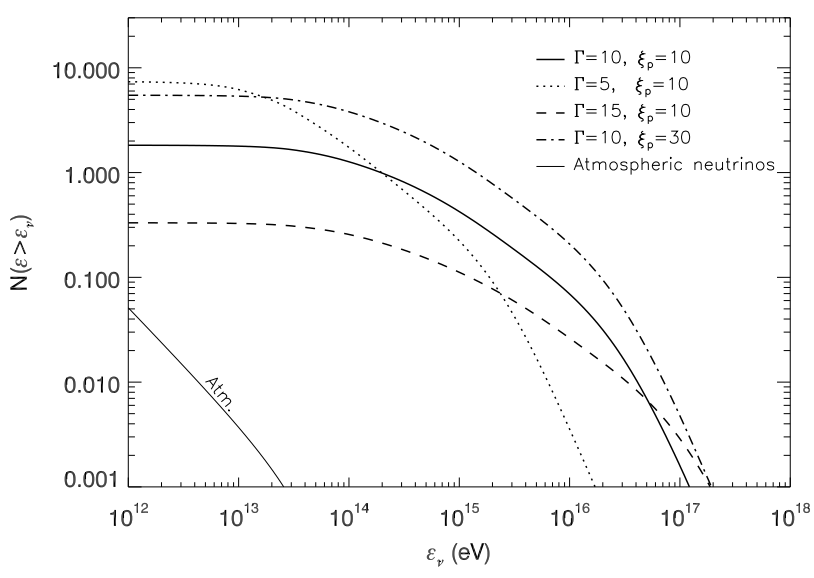

FIG. 2: The expected number of neutrinos $\left(\nu_{\mu}+\bar{\nu}_{\mu}\right)$ above a certain energy detected from Sw J1644+57 by $\mathrm{Km}^{3}$-scale neutrino detectors such as ICecube. The thin solid line represent the background atmospheric neutrinos. The same parameters as in Fig.1 are used.

of neutrinos that can be detected. If a similar event to Sw J1644+57 occurs at a closer distance (e.g. at $z=0.2$ ) in future, more neutrinos would be detected as well.

Neutrino detection from TDFs can be assured only if the number of background counts is smaller than one. The number of atmospheric neutrinos above $30 \mathrm{TeV}$ expected in the direction of the source during the flare period is

$$
\begin{aligned}
& N_{a t m}\left(>10^{4.5} \mathrm{GeV}\right)=\int_{10^{4.5} \mathrm{GeV}}^{\infty} d \varepsilon_{\nu} \int d \Omega \int d t \frac{F_{\nu}^{a t m}}{\varepsilon_{\nu}^{2}} P_{\nu \mu} A \\
& \simeq 3 \times 10^{-3}\left(\frac{\Delta T}{10^{6} \mathrm{~s}}\right) A_{10}\left(\frac{\theta}{1^{\circ}}\right)^{2},
\end{aligned}
$$

where $\Delta T$ is characteristic duration of the TDF, $\theta \simeq$ $0.5^{\circ}-0.6^{\circ}$ is the angular resolution of the neutrino detector at $30 \mathrm{TeV}-\mathrm{PeV}[22], F_{\nu}^{a t m}$ is the cosmic-ray induced atmospheric neutrino background flux. We fit the atmospheric neutrino data measured by Icecube [23] with a single power-law function, which gives $F_{\nu}^{a t m} \simeq$ $4.7 \times 10^{-8} \mathrm{ergcm}^{-2} \mathrm{~s}^{-1} \mathrm{sr}^{-1}\left(\varepsilon_{\nu} / 1 \mathrm{TeV}\right)^{-\beta}$ with $\beta \simeq 1.44 \mathrm{in}$ the energy range of $0.1-400 \mathrm{TeV}$. Since the number of atmospheric neutrinos above $30 \mathrm{TeV}$ expected in the direc- tion of the source during the flare period is much smaller than one, a detection of two neutrinos at energies above $30 \mathrm{TeV}$ from TDF sources will be highly significant.

Discussions. - Neutrino emission has been predicted to be produced by relativistic jets in gammaray bursts [11, 17, 24], active galactic nuclei [25] and microquasars [26]. Observations of Sw 1644+57 suggest that powerful jets are formed in TDFs, which have larger fluence in $\mathrm{X} / \gamma$-rays than the brightest GRBs and have higher X-ray luminosity than AGNs. There are three factors that are favorable for bright neutrino emission produced in such TDFs: 1) large fluence in the X-ray emission, which suggests large fluence in accelerated protons; 2) high pion production efficiency as implied by the high opacity of high-energy gamma-rays, which leads to a high fraction of the proton energy lost into secondary pions; 3) insignificant radiative cooling of secondary pions and muons, which leads to an almost flat neutrino spectrum up to $\sim 10^{16} \mathrm{eV}$. The main uncertainty lies in the ratio between the energy density of protons and the energy density in X-rays. In the magnetic field-dominated jet model, the proton energy density is subdominant, so the neutrino flux would be low, whereas in the matterdominated jet model, we expect one to several neutrinos detectable by $\mathrm{Km}^{3}$-scale neutrino detectors from TDFs similar to Sw $1644+57$. The Swift BAT, with a field of view of $4 \pi / 7 \mathrm{sr}$, has detected one TDF in 7 years, so the all-sky rate of TDFs would be one event in every one year. For neutrino detectors such as Icecube that has a $2 \pi \mathrm{sr}$ field of view, we expect one TDF event in the field of view of Icecube every two years, if the electro-magnetic counterparts can be identified. Therefore neutrino observations provide a promising approach to diagnose the composition of the jets resulted from tidal disruption of stars by massive black holes in the galaxy center.

This work is supported by the NSFC under grants 10973008,10873009 and 11033002 , the 973 program under grants 2009CB824800 and 2007CB815404, the Program for New Century Excellent Talents in University, the Qing Lan Project and the Fok Ying Tung Education Foundation. KSC is supported by a GRF Grant of the Government of the Hong Kong SAR under HKU 7011/10P.
[1] M. J. Rees, Nature 333, 523 (1988); C. R. Evans, C. S. Kochanek, ApJL 346, L13 (1989); J. Wang, D. Merritt, ApJ 600, 149 (2004); L. E. Strubbe, E. Quataert, MNRAS 400, 2070 (2009).

[2] S. Komossa, J. Greiner, A\&A 349, L45 (1999);S. Gezari, et al., ApJ 676, 944 (2008); S. van Velzen, et al., arXiv:1009.1627 (2010).

[3] K. S. Cheng \& Y. Lu, MNRAS, 320, 235 (2001); K. S. Cheng, et al., ApJ, 645, 1138 (2006); K. S. Cheng, et al., A\&A, 473, 351 (2007); D. Giannios, B. D. Metzger, arXiv:1102.1429 (2011).
[4] D. Burrows, et al., Nature, submitted, arXiv:1104.4787

[5] J. Bloom, et al., Science, 333, 203 (2011)

[6] A. Levan, et al. Science, 333, 199 (2011)

[7] U. Barres de Almeida \& A. De Angelis, arXiv:1104.2528 (2011); L. Shao, et al., ApJ Letters, accepted, arXiv:1104.4685 (2011); J. K. Cannizzo, et al. arXiv:1105.2816 (2011); A. Socrates, arXiv:1105.2557 (2011)

[8] A. Zauderer, et al. Nature, submitted (2011)

[9] P. Mészáros, \& M.J. Rees, Mon. Not. Roy. Astron. Soc. 269, 41P (1994); B. Paczyński \& G. Xu, Astrophys. J. 
427, 708 (1994). M. Spada, G. Ghisellini, D. Lazzati, \& A. Celotti, MNRAS, 325, 1559 (2001); Böttcher, M. \& Dermer, C. D., Astrophys.J., 711, 445 (2010)

[10] G. R. Farrar \& A. Gruzinov, Astrophys.J., 693, 329 (2009)

[11] K. Murase \& S. Nagataki, Phys.Rev.D 73063002 (2006); K. Murase \& S. Nagataki, Phys. Rev. Lett., 97051101 (2006)

[12] A. Panaitescu, P. Kumar, Astrophys.J. 560, L49 (2001)

[13] B. Katz, R. Budnik, \& E. Waxman, JCAP, 3, 20 (2009); S.T. Scully \& F. W. Stecker, Astropart. Phys., 16271 (2002); V. Berezinsky, Adv. In Space Res. 41, 2071 (2008)

[14] F. W. Stecker, Phys. Rev. Lett. 21, 1016 (1968)

[15] A. Mücke, et al., Pub. Astron. Soc. Australia, 16, 160 (1999)

[16] A. M. Atoyan \& C. D. Dermer, 2003, ApJ, 586, 79

[17] E. Waxman, J. Bahcall, Phys. Rev. Lett., 78, 2292 (1997)

[18] J.P. Rachen, and P. Mészáros, Phys. Rev. D, 58, 123005 (1998)

[19] S. Razzaque, P. Mészáros \& E. Waxman, Phys. Rev. Lett., 93, 181101 (2004)
[20] A. Mücke et al., Nucl. Phys. B. Proc. Suppl., 80 (2000)

[21] T. K. Gaisser, F. Halzen, and T. Stanev, Phys. Repts. 258(3), 173 (1995); S. Razzaque and P. Mészáros, and E. Waxman, Phys. Rev. D, 69, 023001 (2004); K. Ioka, et al. ApJ, 633, 1013(2005)

[22] J. Ahrens, , et al. Astropart. Phys., 20, 507 (2004)

[23] R. Abassi et al., Phys. Rev. D, 83, 012001 (2011).

[24] C. D. Dermer, \& A. Atoyan, Phys. Rev. Lett., 91, 071102 (2003); S. Razzaque, P. Mészáros \& E. Waxman 2003, Phys. Rev. Lett., 90, 241103; X. Y. Wang \& Z. G. Dai, ApJ, 691, L67 (2009)

[25] K. Mannheim, T. Stanev, and P.L. Biermann, Astron. Astrophys. 260, L1 (1992); C. D. Dermer, \& A. Atoyan, Phys. Rev. Lett.,87, 221102 (2001); F. W. Stecker, Phys. Rev. D, 72, 107301 (2005); J. K. Becker \& P. L. Biermann, Astroparticle Physics, 31, 138 (2009)

[26] A. Levinson, \& E. Waxman, Phys. Rev. Lett., 87, 171101 (2001); F. Aharonian, et al., Journal of Physics: Conference Series, 39, 408 (2006). 